\title{
Spectral self-imaging effect by time-domain multilevel phase modulation of a periodic pulse train
}

\author{
José Caraquitena, ${ }^{1,3}$ Marta Beltrán, ${ }^{1, *}$ Roberto Llorente, ${ }^{1}$ Javier Martí, ${ }^{1}$ and Miguel A. Muriel ${ }^{2}$ \\ ${ }^{1}$ Nanophotonics Technology Center, Universidad Politécnica de Valencia, 46022 Valencia, Spain \\ ${ }^{2}$ Escuela Técnica Superior de Ingenieros de Telecomunicación (E.T.S.I. Telecomunicación), \\ Universidad Politécnica de Madrid, 28040 Madrid, Spain \\ ${ }^{3}$ e-mail: jcaraqui@ntc.upv.es \\ *Corresponding author: mbeltran@ntc.upv.es
}

Received May 25, 2010; revised October 5, 2010; accepted October 10, 2010; posted February 9, 2011 (Doc. ID 129019); published March 9, 2011

\begin{abstract}
We propose and analyze a novel (to our knowledge) approach to implement the spectral self-imaging effect of optical frequency combs. The technique is based on time-domain multilevel phase-only modulation of a periodic optical pulse train. The method admits both infinite- and finite-duration periodic pulse sequences. We show that the fractional spectral self-imaging effect allows one to reduce by an integer factor the comb frequency spacing. Numerical simulation results support our theoretical analysis. (c) 2011 Optical Society of America

OCIS codes: $\quad 070.6760,320.5540,060.4080,320.1590,320.7085$.
\end{abstract}

The temporal self-imaging effect, or temporal Talbot effect, occurs when periodic trains of transform-limited optical pulses with repetition period $T_{\text {rep }}$ propagate through a quadratic phase-only filter, e. g., a dispersive medium in a first-order approximation. An appropriate amount of dispersion, given by the so-called Talbot condition, leads either to reproduction of the original pulse train (integer temporal Talbot effect) [1] or to repetition-rate multiplication by an integer factor (fractional temporal Talbot effect) [2], as shown in Fig. 1(a). In addition, for other well-known dispersion values, a temporal delay equal to half of the period of the output pulse train is obtained (inverted Talbot effect). Traditional implementations of the temporal Talbot effect include standard single-mode fibers [3] and linearly chirped fiber Bragg gratings [4]. In the frequency domain, a periodic pulse train is described by an optical frequency comb with frequency spacing equal to the pulse repetition rate, i.e., $f_{\text {rep }}=1 / T_{\text {rep }}$, as shown in Fig. 1(a).

Similarly, the spectral self-imaging effect, or spectral Talbot effect, occurs when a periodic sequence of pulses is globally chirped by an optical phase-only modulator with a quadratic time-domain response. Under specific temporal chirping conditions, the comb frequency spacing is reduced by an integer factor while the comb envelope is unchanged, as shown in Fig. 2(a). For other chirping conditions, a frequency shifting effect is observed in the frequency comb. The first proposal on these spectral Talbot effects [5] assumed a particular chirping mechanism based on cross-phase modulation with a long Gaussian pump pulse. This approach, however, only admits periodic pulse sequences with limited time duration, due to the intrinsic finite extent of the chirping effect produced by the long Gaussian pump pulse.

In this Letter, we propose a novel approach for the practical implementation of the spectral self-imaging effect. The technique requires the time-domain multishift phase modulation of an input pulse train. This phenomenon exhibits interesting features, including tunability in comb frequency spacing and the ability of comb fre- quency shifting, which can find practical application in optical communications [] ] and optical signal processing [7]. Our theoretical proposal is confirmed by numerical simulations.

First, let us assume the propagation of a periodic pulse train through a general quadratic phase-only optical filter, described by the spectral transfer function $H(\omega)=$ $\exp \left(i \Phi_{2} \omega^{2} / 2\right)$, where $\Phi_{2}$ is the so-called group delay dispersion coefficient [2]. When the value of $\Phi_{2}$ verifies the well-known temporal Talbot condition [2],

$$
\Phi_{2}= \pm \frac{s}{r} \frac{2 \pi}{\omega_{\text {rep }}^{2}},
$$

an undistorted and multiplied copy of the initial pulse train is obtained. In this expression, $s$ and $r$ are mutually prime integer numbers, and $\omega_{\text {rep }}=2 \pi f_{\text {rep }}$ is the repetition rate of the original pulse train. The pulse multiplication factor is given by the integer $r$. In Fig. 1(a), we schematically show an example of the temporal Talbot effect with $r=2$.
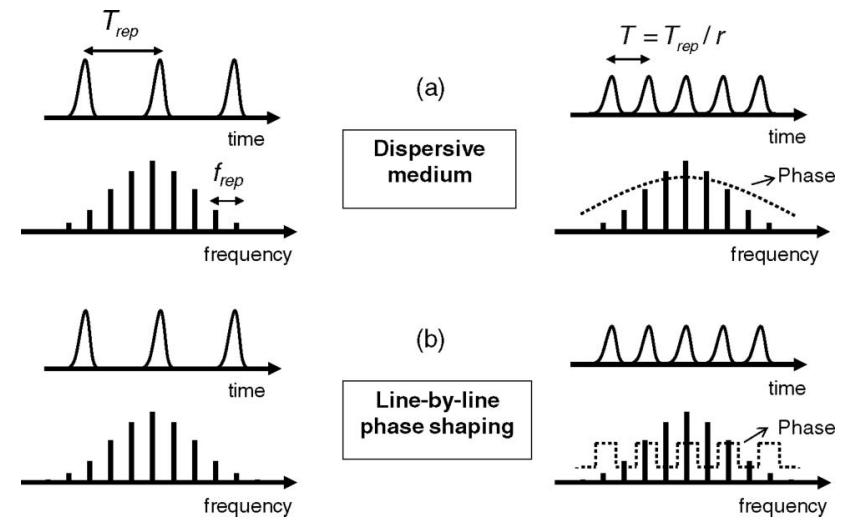

Fig. 1. Illustration of the temporal Talbot effect using (a) a quadratic phase-only filter and (b) line-by-line phase-only filtering. In both cases, two-times repetition-rate multiplication is shown, i.e., $r=2$ [Eqs. (1) and (2), respectively]. 

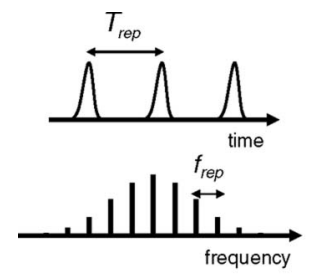

(a)
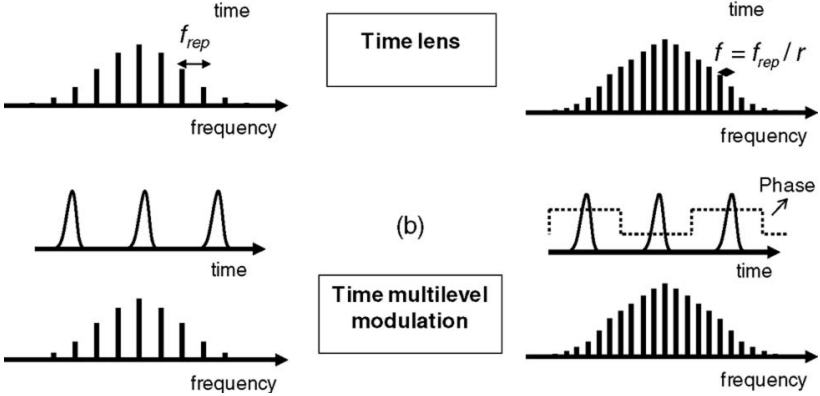

frequency

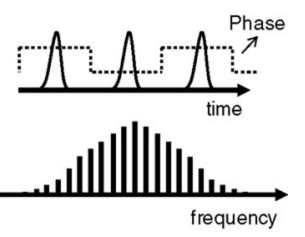

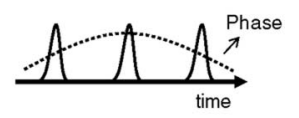

Fig. 2. Illustration of the spectral Talbot effect (a) using quadratic phase modulation in the time domain (time lens) and (b) by multilevel time phase modulation. In both examples, $r=$ 2 [Eqs. (3) and (4), respectively], so that the frequency spacing is reduced by a factor of 2 .

It is important to note that the application of continuous quadratic phase filtering is only a particular option to obtain the temporal Talbot effect. In general, there exists a family of periodic Talbot filters [8] for pulse repetitionrate multiplication. In addition, continuous quadratic phase filtering is unnecessary to obtain the temporal Talbot effect. In fact, only the spectral phases at frequencies equal to the discrete spectral lines of the frequency comb, $\omega_{n}=\omega_{0}+n \omega_{\text {rep }}$, are relevant, where $\omega_{0}$ is the optical carrier frequency and $n$ is an integer. The Talbot condition [Eq. (1)] provides the phase shifts that must be applied to the different spectral lines to obtain repetitionrate multiplication [9]:

$$
\phi\left(\omega_{n}\right)= \pm \frac{s}{r} \pi n^{2} .
$$

Individual control of the phase on each spectral comb component can be achieved by using the so-called spectral line-by-line shaping approach [10]. A traditional implementation of line-by-line shaping involves spectral (de)multiplexing with a diffraction grating [10] and phase control with arrayed modulators [11]. In practice, the actual phase shifts in Eq. (2) are usually applied modulo $2 \pi$, which yields a periodic Talbot phase filter. This line-byline phase-filtering technique constitutes an alternative approach to implement the temporal Talbot effect, as reported in [9]. Figure 1(b) illustrates the case of two-times repetition-rate multiplication by applying the periodic phase filter $\{0, \pi / 2,0, \pi / 2, \ldots\}$, which results from Eq. (2) with $s=1, r=2$. We note that only the intensity profile is multiplied, and the output train exhibits a pulse-to-pulse phase variation, not shown in the figure.

By performing a heuristic analysis, we now investigate the Talbot effect in frequency domain. If the time and spectrum domains are exchanged, it is clear from the above theory that the spectral counterpart of the standard temporal Talbot effect occurs when a periodic pulse train is globally chirped by a quadratic temporal phase modulation, i.e., $h(t)=\exp \left(i \varphi t^{2}\right)$, where $\varphi$ is the chirp parameter, as shown in Fig. 2(a). The Talbot condition on the chirp parameter is directly obtained from Eq. (1), resulting in the expression

$$
\varphi= \pm \frac{s}{r} \frac{\omega_{\mathrm{rep}}^{2}}{4 \pi},
$$

where $s$ and $r$ are again mutually prime integer numbers. In the framework of the space-time duality [12], the effect of the temporal chirp is associated with the action of a time lens. In practice, this chirping effect can be achieved using, for example, electro-optic modulation [13] or fourwave mixing [14] . The reported condition (3) constitutes a generalization of the result derived by Azaña [5] for a particular time-lens mechanism based on cross-phase modulation. Of course, our condition [Eq. (3)] reproduces the result in [5] when the chirp coefficient $\varphi$ is expressed in terms of the parameters involved in the cross-phase modulation process, e. g., the pump peak power and the nonlinear medium coefficient.

Similarly, again by exchanging the time and frequency domains, it is straightforward to conclude that an appropriate time-domain periodic multistep phase modulation of a pulse train also leads to the spectral self-imaging effect. The phase shifts are directly derived from Eq. (2), yielding

$$
\varphi_{n}= \pm \frac{s}{r} \pi n^{2},
$$

where $\varphi_{n}$ is the phase shift applied on the pulse $n$ in the sequence. These phase shifts can be assumed to be applied on time slots equal to the pulse repetition rate $T_{\text {rep. }}$. In practice, however, it would be sufficient to guarantee that the same phase shift is applied along the whole pulse duration. If these phase shifts are reduced to a $2 \pi$ range, a periodic sequence of phase steps is obtained. A schematic diagram of the spectral Talbot effect by the proposed alternative approach based on time-domain multiphase modulation is sketched in Fig. 2(b), where the case $s=1, r=2$ in Eq. (4) is assumed so that a decrease in the comb spacing by a factor of 2 is obtained.

We have performed several numerical simulations to validate the theory on the spectral Talbot effect by multiphase time modulation. In particular, we first assume a periodic infinite sequence of 5 ps FWHM Gaussian pulses with repetition rate $f_{\text {rep }}=10 \mathrm{GHz}$, as shown in Fig. 3(a). In Fig. 3(b) we show the corresponding comblike frequency spectrum. Figure 3(c) shows the output spectral comb obtained with the inverted integer spectral Talbot effect at $s=1, r=1$ in Eq. (4) so that the periodic phase modulation $\{0, \pi, 0, \pi, \ldots\}$ is applied on the input pulse train. Note that a comb frequency shifting by half of the line spacing is obtained. As a second case, in Fig. 3(d) we show the output comb that results from the periodic time-domain modulation $\{0, \pi / 2,0, \pi / 2, \ldots\}$, as derived from Eq. (4) with $s=1, r=2$. In this case, the comb frequency spacing is reduced by a factor of 2 . The introduced approach for spectral Talbot effect implementation also allows for input pulse sequences with finite duration. In particular, we have performed similar simulations assuming an input pulse sequence constituted by only 10 pulses with random amplitude noise and the same pulse width and repetition rate as in the previous example. The corresponding frequency comb is similar to that shown in Fig. 3(b), but now, as expected, we find a finite linewidth in the spectral lines, which is roughly determined by the whole time duration of the input 

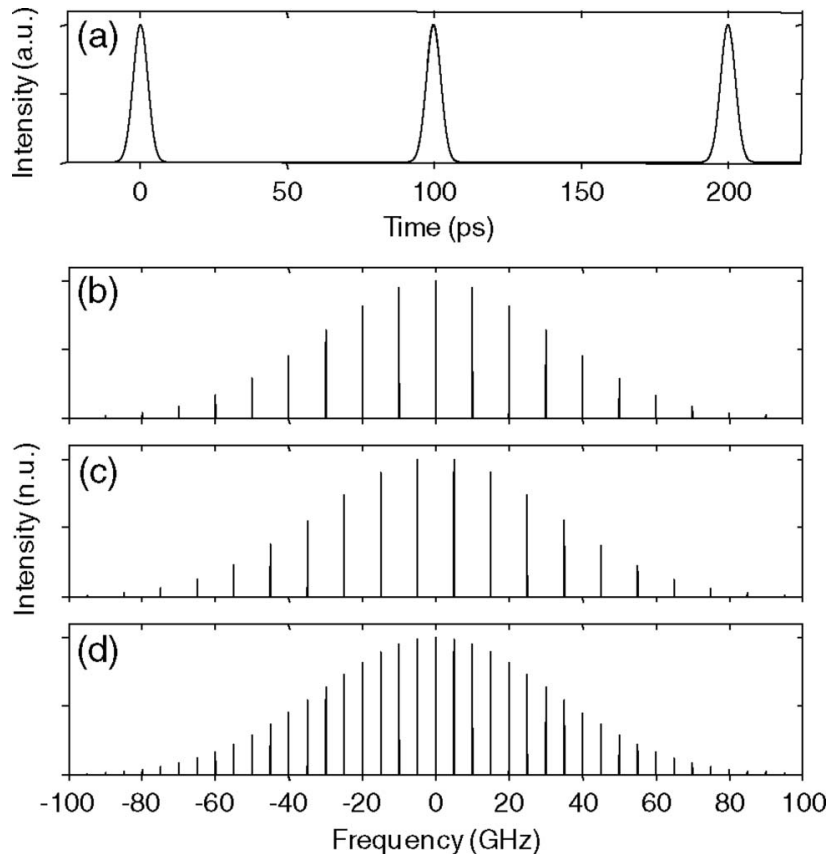

Fig. 3. Results from the simulation of the spectral Talbot effect with infinite duration pulse trains: (a) input pulse train, (b) input frequency comb, (c) output frequency comb after time-domain modulation with $s=1, r=1$ in Eq. ()ㅡ), obtaining a comb shift by half of a period, (d) output frequency comb after time-domain modulation assuming $s=1, r=2$ in Eq. (4).

sequence [5]. With the same spectral periodic filters previously introduced for an infinite pulse train, we again obtain a frequency comb shifting effect and a decrease in the frequency spacing. Additional simulations reveal that the resultant comb envelope is insensitive to the amplitude noise in the input sequence. However, the side lobes found in the output spectral lines are highly dependent on both the whole time duration and amplitude noise of the input sequence. Furthermore, additional numerical simulations show that inaccuracies in the phase shifts translate into nonuniformity in the resultant comb envelope.

Note that only biphase sequences are involved in the introduced examples, so on-off keying modulation techniques should be appropriate for the practical implementation of these examples. In general, however, a more complex multilevel phase modulation is required for the implementation of the spectral Talbot effect. For instance, when $s=1, r=4$, the periodic sequence $\{0, \pi / 4, \pi, \pi / 4, \ldots\}$, according to Eq. (4), must be applied for achievement of a fourfold decrease in the comb line spacing. A simple method to implement the proposed ap- proach can be to use electro-optic phase modulation, e. g., advanced phase modulation techniques such as differential phase shift keying modulators [15]. However, current modulators could only process frequency combs with pulse repetition rates lower than $\sim 50 \mathrm{GHz}$. For higher rates, nonlinear optical processes could be applied, as suggested in [5].

In conclusion, a simple approach for the implementation of the spectral self-imaging effect has been proposed and analyzed. The technique requires the phase multishift time modulation of an input periodic optical pulse train of either infinite or finite duration. The use of this effect on frequency combs may be relevant for the development of multiwavelength sources with reconfigurable frequency spacing and offset.

This work has been supported by the Generalitat Valenciana (Grant GV/2009/044), by the Vicerrectorado de Investigación, Universidad Politécnica de Valencia (Grant PAID-06-08/3276) and by the Ministerio de Ciencia e Innovación of Spain under Project "Plan Nacional de I +D+I TEC2007-68065-C03-02”. J. Caraquitena and M. Beltrán also acknowledge financial support from the Ministerio de Ciencia e Innovación through the "Juan de la Cierva" research program and FPI Grant BES-200612066, respectively.

\section{References}

1. T. Jannson and J. Jannson, J. Opt. Soc. Am. 71, 1373 (1981).

2. J. Azaña and M. A. Muriel, Opt. Lett. 24, 1672 (1999).

3. S. Arahira, S. Kutsuzawa, Y. Matsui, D. Kunimatsu, and Y. Ogawa, J. Lightwave Technol. 16, 405 (1998).

4. S. Longhi, M. Marano, P. Laporta, O. Svelto, M. Belmonte, B. Agogliati, L. Arcangeli, V. Pruneri, M. N. Zervas, and M. Ibsen, Opt. Lett. 25, 1481 (2000).

5. J. Azaña, Opt. Lett. 30, 227 (2005).

6. A. Bellemare, M. Karasek, M. Rochette, S. LaRochelle, and M. Tetu, J. Lightwave Technol. 18, 825 (2000).

7. J. Caraquitena and J. Martí, Opt. Lett. 34, 2084 (2009).

8. J. Azaña and S. Gupta, Opt. Express 14, 4270 (2006).

9. J. Caraquitena, Z. Jiang, D. E. Leaird, and A. M. Weiner, Opt. Lett. 32, 716 (2007).

10. Z. Jiang, C.-B. Huang, D. E. Leaird, and A. M. Weiner, Nat. Photon. 1, 463 (2007).

11. A. M. Weiner, Rev. Sci. Instrum. 71, 1929 (2000).

12. B. H. Kolner, IEEE J. Quantum Electron. 30, 1951 (1994).

13. A. A. Godil, B. A. Auld, and D. M. Bloom, IEEE J. Quantum Electron. 30, 827 (1994).

14. R. Salem, M. A. Foster, A. C. Turner, D. F. Geraghty, M. Lipson, and A. L. Gaeta, Opt. Lett. 33, 1047 (2008).

15. P. J. Winzer and R. J. Essiambre, J. Lightwave Technol. 24, 4711 (2006). 